
ИНДУЦИРОВАННОГО ТИРЕОТОКСИКОЗА И РАКА ПИЩЕВОДА

() А.С. Кузьмичев ${ }^{1 *}$, К.В. Павелец ${ }^{1}$, А.Л. Акинчев ${ }^{1}$, М.А. Протченков ${ }^{2}$, В.И. Ломакин ${ }^{2}$, М.К. Павелец ${ }^{2}$, С.А. Калюжный ${ }^{2}$, E.В. Шепичев ${ }^{2}$

'Санкт-Петербургский государственный педиатрический медицинский университет», Санкт-Петербург, Россия ${ }^{2}$ СПбГБУЗ «Городская Мариинская больница», Санкт-Петербург, Россия

В статье представлен успешный опыт хирургического лечения больного со злокачественной опухолью пищевода, которая была выявлена на фоне амиодарон-индуцированного тиреотоксикоза. Тиреоидэктомия позволила быстро устранить тиреотоксикоз и своевременно выполнить радикальную операцию по поводу рака пищевода.

КЛЮЧЕВЫЕ СЛОВА: амиодарон-индуцированный тиреотоксикоз; щитовидная железа; тиреоидэктомия; рак пищевода; одномоментная резекция пищевода.

\title{
SURGICAL TREATMENT OF PATIENT WITH COMBINATION OF AMIODARON-INDUCED THYROTOXIC AND ESOPHAGESL CANCER
}

(c) Alexander S. Kuzmichev ${ }^{1 *}$, Konstantin V. Pavelets ${ }^{1}$, Alexey L. Akinchev ${ }^{1}$, Mikhail A. Protchenkov², Vasiliy I. Lomakin², Mikhail K. Pavelets², Sergey A. Kalyuzhny², Evgeniy V. Shepichev ${ }^{2}$

'St. Petersburg State Pediatric Medical University, St. Petersburg, Russia

${ }^{2}$ Mariinsky City Hospital, St. Petersburg, Russia

The article presents the successful experience of surgical treatment of a patient with a malignant tumor of the esophagus, which was detected in a patient with amiodarone-induced thyrotoxicosis. Thyroidectomy made it possible to quickly eliminate thyrotoxicosis and timely perform radical surgery for esophageal cancer.

KEYWORDS: amiodarone-induced thyrotoxicosis; thyroid gland; thyroidectomy; cancer of the esophagus; simultaneous resection of the esophagus.

\section{ВВЕДЕНИЕ}

Амиодарон (Ам) используется для лечения опасных для жизни аритмий при отсутствии эффекта от других антиаритмических препаратов [1]. Он положительно влияет на сердечно-сосудистую систему, но способен оказывать отрицательное воздействие на функцию щитовидной железы (ЩЖ), что ограничивает его применение [2]. Известно, что при метаболизме амиодарона высвобождается 6-9 мг йода в сутки, что превышает суточную потребность в десятки раз. В случае длительного применения создается тканевое депо. Нарушение тиреоидного статуса, в частности тиреотоксикоз, способно оказывать проаритмогенное влияние, ухудшить течение аритмии и быть опасным для жизни пациента [3]. Патогенез амиодарон-индуцированного тиреотоксикоза (АИТ) до сих пор до конца не выяснен. Выделяют АИТ 1-го и 2-го типов, а также смешанную форму, сочетающую два различных процесса в клетках щж [4]. При исходной патологии щЖ йод, высвобождаемый из препарата, увеличивает синтез тиреоидных гормонов в зонах автономии или запускает аутоиммунный процесс, вследствие чего развивается АИТ 1-го типа. У пациентов с интактной ЩЖ Ам, обладающий цитотоксическим влиянием, вызывает деструкцию ЩЖ, что приводит к развитию АИТ 2-го типа и выходу ранее синтезированных тиреоидных гормонов в кровоток. При наличии двух процессов развивается тиреотоксикоз смешанного типа.
При длительном назначении Ам дисфункция ЩЖ развивается у 15-20\% пациентов. Развитие дисфункции может наступить во время лечения и после отмены терапии Ам. Поэтому мониторинг функции ЩЖ необходимо проводить до лечения, каждые 6 мес во время лечения и через 6-12 мес после отмены препарата. АИТ 1-го типа развивается в среднем через 2-6 мес после назначения Ам, АИТ 2-го типа - через 27-32 мес, что требует более длительного наблюдения после отмены препарата [5]. При жизненно угрожающих аритмиях быстрое восстановление эутиреоза может быть достигнуто сеансами плазмафереза с последующей тиреоидэктомией [6]. Однако тиреоидэктомия как метод лечения АИТ используется редко. Так, по данным клиники Мауо, с 2002 по 2016 гг. из 350 больных с данной патологией хирургическое лечение было проведено 17 больным. Авторы отмечают высокий процент послеоперационных осложнений. На 17 тиреоидэктомий зарегистрировано 7 осложнений, потребовавших повторной госпитализации с одним летальным исходом [7].

В России стандартом хирургического лечения рака пищевода (РП) является расширенная операция из комбинированного доступа с выполнением двухзональной лимфодиссекции в средостении до верхней апертуры грудной клетки, в брюшной полости - в объеме $\mathrm{D}_{2}$, как при раке желудка с наложением внутриплеврального анастомоза [8, 9]. Цель работы - продемонстрировать возможность успешного хирургического лечения РП у больного с АИТ. 
Пациент П., 48 лет, поступил в хирургическое отделение Мариинской больницы г. Санкт-Петербурга 17.11.18 г. с жалобами на слабость, недомогание, сердцебиение, перебои в работе сердца, одышку. Из анамнеза: в 2016 г. перенес острое нарушение мозгового кровообращение по ишемическому типу. В связи с выявленными пароксизмами мерцательной аритмии больному, наряду с базовой терапией, назначен кордарон, который он принимал с сентября 2017 г. по февраль 2018 г. В январе 2018 г. больной обнаружил увеличение шейных лимфатических узлов слева. В течение нескольких месяцев проходил обследование по поводу неспецифической лимфаденопатии с вовлечением шейных, подмышечных, средостенных, забрюшинных, паховых лимфоузлов. В сентябре больной госпитализирован в отделение онкогематологии Ленинградской областной клинической больницы. В процессе обследования при фиброгастродуоденоскопии выявлен рак средней трети пищевода. Гистологическая картина дифференцированного инвазивного плоскоклеточного рака. В связи с пароксизмами фибрилляции предсердий (тахисистолическая форма) больному проведено исследование тиреоидного статуса (табл. 1). Диагностирован АИТ смешанного типа, по поводу которого назначена комбинированная терапия тиреостатиком и глюкокортикоидами (ГК).

На момент поступления в хирургическое отделение пациент получал тирозол 40 мг/сут, преднизолон 30 мг/сут, конкор 5 мг, амлодипин 10 мг, эликвис 5 мг. Из-за отсутствия на момент госпитализации результатов лабораторно-инструментальных исследований тип тиреотоксикоза определен не был. Несмотря на проводимую терапию, у пациента сохранялись пароксизмы фибрилляции предсердий.

При ультразвуковом исследовании ЖЩ выявлено узловое образование левой доли 10 мм. Выполнена тонкоигольная аспирационная биопсия: цитологическая картина фолликулярной опухоли.

Объективно: состояние удовлетворительное, кожные покровы и слизистые физиологической окраски, повышенной влажности на ощупь. Дыхание жесткое, хрипов нет. Тоны сердца приглушенные. Частота сердечных сокращений - до 90 в минуту. ЩЖ при пальпации безболезненная, узловые образования не выявлены. Лабораторное исследование антител к рецепторам тиреотропного гормона (проведено впервые) - уровень антител 0,30 ME/л (N 0,00-1,75). Сцинтиграфия ЩЖ с Tc99m - изображение очень низкой контрастности из-за выраженного снижения уровня накопления радиофармпрепарата (РФП), возможно, обусловленного приемом тиреостатиков.

На основании анализа полученных данных (нормальный уровень антител, снижение накопления РФП) был сделан вывод о наличии у пациента АИТ 2-го типа. Несмо- тря на проводимую комбинированную терапию тиреостатиками и ГК, в течение 4 мес явления тиреотоксикоза не купировались, пароксизмы мерцательной аритмии участились, перейдя в постоянную форму. Учитывая неэффективность проводимой терапии и необходимость хирургического лечения РП, было решено для устранения тиреотоксикоза выполнить больному тиреоидэктомию. Предоперационная подготовка заключалась в проведении двух сеансов плазмафереза, отмене антикоагулянтов, внутривенном введении препаратов калия и гликозидов. В плановом порядке под эндотрахеальным наркозом 06.12.2018 больному выполнена тиреоидэктомия. Через 2 ч после операции у больного восстановился синусовый ритм. Течение послеоперационного периода без особенностей. Выписан на 5-е сутки после операции на заместительной терапии L-тироксином. Гистологическое заключение: диффузно-узловой фолликулярный зоб.

В дальнейшем пациент поступил в плановом порядке в 6-е хирургическое отделение Мариинской больницы для проведения хирургического лечения злокачественного новообразования средней трети грудного отдела пищевода. 10.01.2019 г. выполнена операция в объеме одномоментной резекции пищевода с пластикой желудочным трансплантатом, лимфодиссекцией в объеме $\mathrm{D}_{2}$ и внутривенной фотодинамической терапией с облучением зон лимфодиссекции. На 14-е сутки отмечена отрицательная динамика в виде нарастания подкожной эмфиземы лица, шеи. Проведена гибкая бронхоскопия, в ходе которой выявлен дефект мембранозной части трахеи размерами 0,6×0,3 см над бифуркацией трахеи. В связи с полученными данными больному в экстренном порядке выполнена медиастинотомия с последующим использованием аппарата активной аспирации. Отмечена положительная динамика в виде регресса признаков эмфиземы и стабилизации состояния пациента. На фоне проводимого лечения клинико-инструментальные проявления спонтанного разрыва мембранозной части трахеи полностью купированы.

Гистологическое заключение: аденокарцинома. Гистологический тип клеток - плоскоклеточный, умеренной дифференцировки.

Выписан в удовлетворительном состоянии. Осмотрен через 1 год, состояние больного удовлетворительное. Жалоб не предъявляет.

Таким образом, у данного больного была определена тактика лечения с учетом имеющихся заболеваний и осложнений. Наличие тяжелого тиреотоксикоза, осложненного фибрилляцией предсердий, исключало возможность хирургического лечения РП. В то же время операции по поводу АИТ в мировой практике обычно не являются методом выбора [7]. Однако необходимость быстрого устранения тиреотоксикоза для уменьшения

Таблица 1. Результаты исследования тиреоидного статуса пациента

\begin{tabular}{lccc}
\hline \multicolumn{1}{c}{ Гормоны } & $\mathbf{3 1 . 0 8 . 1 8}$ & $\mathbf{1 1 . 0 9 . 1 8}$ & $\mathbf{1 9 . 1 1 . 1 8}$ \\
\hline ТЗ, ммоль/л (N 1,0-3,0) & 10,80 & 11,07 & 9,33 \\
Т4, ммоль/л (N 12,30-22,80) & 32,50 & 33,39 & 35,87 \\
ТТГ, мкМЕ/мл (N 0,3-4,2) & $<0,004$ & $<0,005$ & $<0,005$ \\
\hline
\end{tabular}

Примечание. Т3 - трийодтиронин; Т4 - тироксин; ТТГ — тиреотропный гормон. 
риска прогрессирования опухоли пищевода обосновала необходимость начать лечение с тиреоидэктомии. Для подготовки больного к операции был использован плазмаферез [6]. Указанная последовательность действий позволила быстро устранить тиреотоксикоз и в кратчайшие сроки выполнить больному хирургическое вмешательство по поводу РП в соответствии с действующими стандартами [8].

\section{ЗАКЛЮЧЕНИЕ}

1. Тиреоидэктомия в сложной клинической ситуации позволила быстро ликвидировать тиреотоксикоз, вызванный назначением амиодарона.
2. Вторым этапом была выполнена радикальная операция по поводу РП с хорошим отдаленным результатом лечения.

\section{ДОПОЛНИТЕЛЬНАЯ ИНФОРМАЦИЯ}

Согласие пациента. Информированное согласие пациента на публикацию персональной медицинской информации в обезличенной форме было получено.

Конфликт интересов. Авторы декларируют отсутствие явных и потенциальных конфликтов интересов, связанных с публикацией настоящей статьи.

Участие авторов. Все авторы внесли значимый вклад в подготовку статьи, прочли и одобрили финальную версию до публикации.

\section{СПИСОК ЛИТЕРАТУРЫ | REFERENCES}

1. Сердюк С.Е., Бакалов С.А., Соболева В.А., и др. Амиодаронассоциированная дисфункция щитовидной железы: частота развития, возможности коррекции // Кардиология. - 2004. T. 44. - №10. - C. 32-37. [Serdyuk SYe, Bakalov SA, Soboleva VA, et al. Amiodar-associated thyroid dysfunction: developmental frequency, correction possibilities. Cardiology. 2004;44(10):32-37. (In Russ.)].

2. Мельниченко Г.А., Свириденко Н.Ю., Молашенко Н.В., и др. Индуцированная амиодароном дисфункция щитовидной железы (патогенез, диагностика, лечение) // Терапевтический архив. — 2003. - T. 75. - №8. - C. 92-96. [Melnichenko GA, Sviridenko NYu, Molashenko NV, et al. Amyodarone-induced thyroid dysfunction (pathogenesis, diagnosis, treatment). Therapeutic archive. 2003;75(8):92-96. (In Russ.)].

3. Мкртумян А.М., Бирюкова Е.В, Егшатян Л.В., и др. Амиодарониндуцированный тиреотоксикоз 1-го типа: особенности ведения пациентов // Эффективная фармакотерапия. - 2019. - Т. 15. №25. - C. 8-12. [Mkrtumyan AM, Biryukova YV, Yegshatyan LV, et al. Amiodarone-Induced Thyrotoxicosis Type 1: Features of Patient Management. Effective farmacotherapy. 2019;15(25):8-12. (In Russ.)]. doi: https://doi.org/10.33978/2307-3586-2019-15-25-8-11

4. Лебедева Е.А., Яблонская Ю.А., Булгакова С.В. Амидарониндуцированный тиреотоксикоз. Современный взгляд на проблему // Клиническая и экспериментальная миреоидология. — 2017. - Т. 13. - №2. - С. 31-38. [Lebedeva EA, lablonskaia IA, Bulgakova SV. Amiodarone- induced thyrotoxicosis: state of the art. Clinical and experimental thyroidology. 2017;13(2):31-38. (In Russ.)]. doi: https://doi.org/10.14341/ket2017231-38

5. Tomisti $L$, Rossi G, Bartalena $L$, et al. The onset time of amiodaroneinduced thyrotoxicosis (AIT) depends on AIT type. Eur J Endocrinol. 2014;171(3):363-368. doi: https://doi.org/10.1530/EJE-14-0267

6. Jabrocka-Hybel A, BednarczukT, Bartalena L, et al. Amiodaron i tarczyca. Endokrynol Pol. 2015;66(2):176-196. doi: https://doi.org/10.5603/EP.2015.0025

7. Kotwal A, Clark J, Lyden M, et al. Thyroidectomy for AmiodaroneInduced Thyrotoxicosis: Mayo Clinic Experience. J Endocr Soc. 2018;2(11):1226-1235. doi: https://doi.org/10.1210/js.2018-00259

8. Минимальные клинические рекомендации Европейского Общества медицинской онкологии (ESMO) / Под ред. Тюляндина С.А., Носова Д.А., Переводчикова Н.И. М.: Издательская группа РОНЦ им. Н. Н. Блохина РАМН; 2010.436 с. [Minimum clinical recommendations of the European Society of Medical Oncology (ESMO). Ed by Tyulyandin SA, Nosov DA, Perevodchikova NI. Moscow: Publishing group RONC n.a. N.N. Blokhin RAMS; 2010. 436 p. (In Russ.)].

9. Кавайкин Д., Чичеватов Д. Профилактика бронхолегочных осложнений после внутриплевральной эзофагопластики при раке пищевода // Врач. - 2012. — №10. — C. 78-80. [Kavaykin D, Chichevatov D. Prevention of bronchopulmonary complications after intrapleural esophagoplasty with cancer of the esophagus. Vrach. 2012;10:78-80. (In Russ.)].

\section{ИНФОРМАЦИЯ ОБ АВТОРАХ [AUTHORS INFO]}

*Кузьмичев Александр Сергеевич, д.м.н, профессор, врач-хирург [Alexander S. Kuzmichev, MD, PhD, professor]; адрес: Россия, 194100, г. Санкт-Петербург, ул. Литовская, д. 2 [address: Russia, 194100, St. Petersburg, ul. Litovskaya, 2]; ORCID: 0000-0002-6983-368X; eLibrary SPIN: 8012-3448; e-mail: alkuz02@mail.ru

Акинчев Алексей Львович, к.м.н., доцент, врач-хирург [Alexey L. Akinchev, MD, PhD]; ORCID: 0000-0002-7170-0202; eLibrary SPIN: 7334-0768; e-mail: alakinchev@mail.ru

Павелец Константин Вадимович, д.м.н., профессор, врач-хирург [Konstantin V. Pavelets, MD, PhD, professor]; ORCID: 0000-0002-1921-8427; e-mail: prof.kvp@icloud.com

Протченков Михаил Александрович, к.м.н., врач-хирург [Mikhail A. Protchenkov, MD, PhD]; ORCID: 0000-0002-9733-0377; eLibrary SPIN: 4036-6911; e-mail: cooperit62@gmail.com

Ломакин Василий Иванович, врач-хирург [Vasiliy I. Lomakin, MD]; ORCID: 0000-0001-7197-2024;

e-mail: Lomakin.vasilii@yandex.ru

Павелец Михаил Константинович, врач-хирург [Mikhail K. Pavelets, MD]; ORCID: 0000-0001-9120-1593;

e-mail: paveletsmk@icloud.com

Калюжный Сергей Алексеевич, врач-хирург [Sergey A. Kalyuzhny, MD]; ORCID: 0000-0002-3507-6123;

e-mail: sergey_kalyuzhnyy@rambler.ru

Шепичев Евгений Викторович, врач торакальный хирург [Evgeniy V. Shepichev, MD]; ORCID: 0000-0003-3332-715X; e-mail: dr.shepichev@gmail.com 


\section{ИНФОРМАЦИЯ}

Рукопись получена: 22.01.2021. Одобрена к публикации: 18.05.2021.

\section{ЦИТИРОВАТЬ:}

Кузьмичев А.С., Акинчев А.Л., Павелец К.В., Протченков М.А., Ломакин В.И., Павелец М.К., Калюжный С.А., Шепичев Е.В. Хирургическое лечение больного с сочетанием амиодарон-индуцированного тиреотоксикоза и рака пищевода // Эндокринная хирургия. — 2020. — Т. 14. — №4. — С. 26-29. doi: https://doi.org/10.14341/serg12695

\section{TO CITE THIS ARTICLE:}

Kuzmichev AS, Akinchev AL, Pavelets KV, Protchenkov MA, Lomakin VI, Pavelets MK, Kalyuzhny SA, Shepichev EV. Surgical treatment of patient with combination of amiodaron-induced thyrotoxic and esophagesl cancer. Endocrine surgery. 2020;14(4):26-29. doi: https://doi.org/10.14341/serg12695 